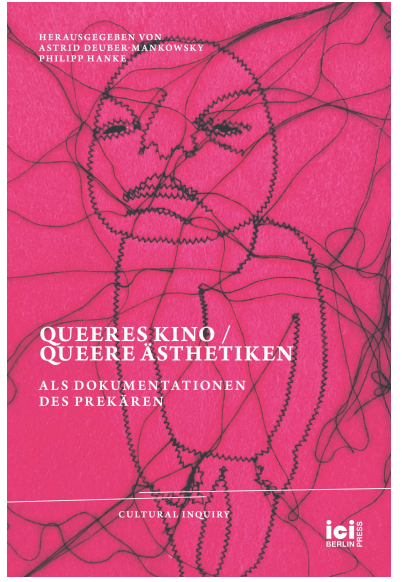

Queeres Kino / Queere Ästhetiken als Dokumentationen des Prekären, hg. v. Astrid Deuber-Mankowsky und Philipp Hanke, Cultural Inquiry, 22 (Berlin: ICI Berlin Press, 2021), S. 69-95
ASTRID DEUBER-MANKOWSKY

\section{Das iPhone als Medium einer queeren Ästhetik und fluiden Subjektivität \\ Charlotte Prodgers BRIDGIT}

ZITIERVORGABE:

Astrid Deuber-Mankowsky, $\gg$ Das iPhone als Medium einer queeren Ästhetik und fluiden Subjektivität. Charlotte Prodgers BRIDGIT«, in Queeres Kino / Queere Ästhetiken als Dokumentationen des Prekären, hg. v. Astrid Deuber-Mankowsky und Philipp Hanke, Cultural Inquiry, 22 (Berlin: ICI Berlin Press, 2021), S. 69-95 <https://doi.org/10.37050/ci-22_04>

ANGABE ZU DEN RECHTEN:

\begin{abstract}
(C) bei den Autor*innen
Dieses Werk ist veröffentlicht unter einer Creative Commons Namensnennung - Weitergabe unter gleichen Bedingungen 4.0 International Lizenz.
\end{abstract}

ABSTRACT: BRIDGIT ist der Titel des iPhone-Videos, mit dem Charlotte Prodger 2018 den Turner Prize gewann. Der Beitrag zeigt, dass Prodger einerseits an bereits etablierte Ästhetiken des experimentellen queeren Kinos anknüpft, andererseits aber auch über diese hinausgeht. Die ästhetische Verschränkung von Körpern, Begehren, Wahrnehmung und Selbstwahrnehmung, Landschaft, Zeit und Queerness ist - auch im Medium des digitalen Filmes - skulptural und setzt darin eine virtuelle Kraft frei, die sich den neoliberalen Normalisierungsprozessen und der Nicht-Intelligibilität von queeren Begehrensformen in subtiler Weise widersetzt.

SCHLAGWÖRTER: Digitale Bildmedien; situierte Subjektivität; fluide Identität; iPhone-Film; queerer Experimentalfilm; queere Kollektivität; transindividuelles Beziehungsgefüge; virtuelle Kraft; lesbisches Begehren; Lesbian Separatism; trans Rights 


\section{Das iPhone als Medium einer queeren Ästhetik und fluiden Subjektivität Charlotte Prodgers BRIDGIT ASTRID DEUBER-MANKOWSKY}

\section{BRIDGIT}

BRIDGIT ist der Titel des 32-minütigen Videos, mit dem die in Schottland lebende bildende Künstlerin Charlotte Prodger 2018 den Turner Prize gewann. ${ }^{1}$ Der Film besteht zur Gänze aus Aufnahmen, die Prodger mit ihrem iPhone drehte, ohne technische Hilfsmittel wie Stativ oder zusätzliche Linsen. BRIDGIT ist der zweite in einer Reihe von Filmen, die in komplexer Weise um Fragen von Identität und Queerness kreisen. Der erste, aus dem Jahr 2015, Stoneymollan Trail, ist eine 43-minütige Kompilation aus Videokamera-Aufnahmen, HD- und iPhone-Footage. ${ }^{2}$ Der jüngste, SaF05, wurde 2019 auf der 58. Biennale in Venedig gezeigt. ${ }^{3}$ In dieser 43-minütigen Videoarbeit zieht Prodger, wie Henriette Gunkel in ihrem Aufsatz in diesem Band detailliert ausführt, Filmszenen aus digitalen Wild-Kameras und Drohnenbilder

1 BRIDGIT, Regie: Charlotte Prodger (Hollybush Gardens, Charlotte Prodger, 2016).

2 Stoneymollan Trail, Regie: Charlotte Prodger (Hollybush Gardens, Charlotte Prodger, 2015).

3 SaF05, Regie: Charlotte Prodger (Hollybush Gardens, Charlotte Prodger, 2019). 
hinzu, um sie über ein explizites Spiel mit ihnen in einen $\gg$ queeren Kontext $\ll$ zu stellen. ${ }^{4}$

Bevor sich Prodger den digitalen Bildmedien zuwandte und Filme als Einkanal-Videos im Kunstraum vorstellte, arbeitete sie mit 16-mm-Film und weiteren analogen Medien und fügte die Abspielgeräte zu Skulpturen zusammen, die sie als Installationen ausstellte. Ihr Interesse galt dem Experimentieren mit der Materialität dieser Techniken und der Erforschung des Verhältnisses von Körper, Technik und der Möglichkeit, die Erfahrung und Wahrnehmung von Körpern durch die Entwicklung neuer, queerer Ästhetiken zu erweitern. Queere Ästhetiken widersetzen sich jenen Normalisierungsprozessen, die Judith Butler 1989 in Das Unbehagen der Geschlechter und 1993 in Körper von Gewicht, im Anschluss an ACT Up und die AIDS-Epidemie und in der Entstehungszeit des New Queer Cinema, so überzeugend als Unsichtbarmachung und als Nicht-Intelligibilität von queeren Begehrensformen und queeren Körpern analysierte. Diese Normalisierungsprozesse stützen und reproduzieren, wie Butler zeigte, nicht nur das System der Heteronormativität, sondern auch die Prekarität von queeren Körpern. Prodger knüpft in BRIDGIT, wie ich im Folgenden zeigen möchte, einerseits an bereits etablierte Ästhetiken des queeren Kinos an, geht aber andererseits auch über diese hinaus. Dabei spielt ihr Interesse am Skulpturalen und an der Materialität der Technik eine zentrale Rolle. Dieses Interesse leitet sie auch im Experimentieren mit dem iPhone als kleine, nah am Körper befindliche, digitale Filmkamera. Die ästhetische Verschränkung von Körpern, Begehren, Wahrnehmung und Selbstwahrnehmung, Landschaft, Zeit und Queerness ist - auch im Medium des digitalen iPhone-Filmes - skulptural und setzt darin eine virtuelle Kraft frei. Dabei verstehe ich diese virtuelle Kraft mit dem Technikphilosophen Gilbert Simondon als eine Kraft des Werdens, die - in Anknüpfung an die Tradition und das Zeitdenken der Transzendentalphilosophie - die Zukunft als Potentialität in die Gegenwart zurücklaufen zu lassen vermag.

4 Vgl. den Beitrag von Henriette Gunkel in diesem Band. 


\section{EIN DENKBILD}

Der Titel des Videos BRIDGIT bezieht sich auf den Namen einer keltischen Göttin, die mit der Kraft des Feuers, des Lichts und mit Fruchtbarkeit verbunden wird, und über eine komplexe Gestalt verfügt. Sie ist unter dem Namen Bridgit bekannt, hatte jedoch wahrscheinlich, wie die anderen neolithischen Gottheiten auch, mehrere einsilbige Namen, also etwa Bride, Brid, Brig oder einfach Bree. Die Namen bilden überhaupt das größte Problem für die Erforschung der Gottheiten in der alten Welt. Sie waren nicht nur an verschiedenen Orten unter verschiedenen Namen bekannt, sondern hatten an demselben Ort auch verschiedene Namen je nach den verschiedenen Phasen ihres Lebensalters: jung, mittel, alt. Die Namen sind zumeist einsilbig, Iterationen, sie bilden einen Rhythmus, fast einen Refrain, ein Ritornell, wenn man sie aufsagt, und scheinen zugleich unverändert seit der Steinzeit.

Man erfährt all diese Informationen über einen Auszug aus Julian Copes Buch The Modern Antiquarian: Pre-Millennial Odyssey through Megalithic Britain. Der Text wird von einer rhythmischen, hellen Stimme in schottischem Englisch zunächst über eine sich vorbeibewegende Landschaft gesprochen, durch die in der Ferne parallel zur Bewegung der Kamera ein roter Lastzug fährt. Die Stimme spricht weiter während wir nach einem Schnitt über ein vom Wind bewegtes Wasser zwei Lastkähne sehen. Sie stehen still, scheinen sich aber zu bewegen, da die Kamera selbst in Bewegung ist - Prodger hat die Szene mit ihrem iPhone offensichtlich von einem sich in Fahrt befindlichen Schiff aus aufgenommen. ${ }^{5}$ Sie hält das iPhone horizontal, so dass das Format 16:9 ist, jenes von Landschaftsaufnahmen. ${ }^{6}$ Man fühlt sich fast ein wenig schwindelig, wie in einem $\mathrm{Zug}$, von dem man nicht weiß, ob er noch steht oder sich schon bewegt. Und man erinnert sich an jenes Denkexperiment, mit dem Galileo Galilei im 17. Jahrhundert, etwas mehr als 270 Jahre vor Einstein, das Relativitätsprinzip formulierte. Es besagt, dass Bewegungen von Körpern nur relativ zu

5 Die Szene kann auf Vimeo eingesehen werden <https://vimeo.com/222200361> [Zugriff: 3. Juni 2021].

6 Vgl. Mason Leaver-Yap, $\gg$ Aesthetics and Anaesthetics in Charlotte Prodgers BRIDGIT «, in Bergen Kunsthall NO.5 (2017) < http://hollybushgardens.co.uk/wp/ wp-content/uploads/CP_Mason-Leaver-Yap_Aesthetics-and-Anaesthetics.pdf> [Zugriff: 3. Juni 2021]. 
Bewegungen anderer Körper festgestellt werden können und nicht zu einem bevorzugten Bezugssystem. Die Szene könnte aufgrund ihrer präzisen formalen Komposition, inhaltlichen Dichte und Dauer im Anschluss an Walter Benjamin als ein »Denkbild « verstanden werden. ${ }^{7} \gg$ Denkbilder $\ll$ erschaffen dichte, situative Bildräume, die, über sich hinausweisend, Denkräume eröffnen. Bereits für Benjamin standen die zu seiner Zeit neuen Medien der technischen Reproduktion, Fotografie und Film, Paten für diese neue Form der Verbindung von Anschauung und Denken.

Charlotte Prodger setzt ihr iPhone, das bei statischen Einstellungen in besonderer Weise das Atmen des Körpers spürbar macht, ein, um mit einer sich konstant in relativer Bewegung zu anderen Bewegungen befindlichen, situierten Subjektivität und fluiden Identität zu experimentieren. So kommt auch die Verbindung zu den keltischen Gottheiten nicht aus dem Nichts, sondern ist mit Erinnerungen verbunden. Sie führt in die Jahre 1992 und 1993 zurück, als Prodger, in der Landschaft im schottischen Aberdeen aufgewachsen, zwischen 18und 19-jährig, kurz vor ihrem Coming-out, Ecstasy-Pillen konsumierte und der Rock-Musiker, Songschreiber, Popstar und Autor Julian Cope wiederholt in ebendiese Landschaft reiste, um die geheimnisvollen, mehr als 4000 Jahre alten Steinkreise aus der Jungsteinzeit zu besuchen, die in Aberdeenshire besonders zahlreich sind und bis dahin wenig beschrieben waren. Julian Cope recherchierte in jener Zeit für sein bereits erwähntes Buch The Modern Antiquarian über die steinzeitlichen Baudenkmäler in Großbritannien, das 1998 mit vielen Bildern neolithisch geprägter Landschaften erschien und schnell überaus populär wurde. ${ }^{8}$

7 Benjamin hat den Begriff $\gg$ Denkbilder $\ll$ als Titel für eine Sammlung von Textminiaturen gewählt. Siehe Walter Benjamin, Gesammelte Schriften, hg. v. Rolf Tiedemann und Hermann Schweppenhäuser, 7 Bde. (Frankfurt a. M.: Suhrkamp, 1972-91), Iv (1972), S. 305-438.

8 Julian Cope, The Modern Antiquarian: Pre-Millennial Odyssey through Megalithic Britain (London: Thorsons, 1998). Im März 2000 wurde die Webseite The Modern Antiquarian als Plattform für die kollaborative Weiterführung der Recherchen von Julian Cope veröffentlicht. Sie wird bis heute genutzt und bespielt und belegt die Popularität, die diese Landschafts- und Geschichtsforschung in Großbritannien im Anschluss an Copes Bildband gewann <https://www.themodernantiquarian.com/home/ $>[\mathrm{Zu}$ griff: 3. Juni 2021]. 


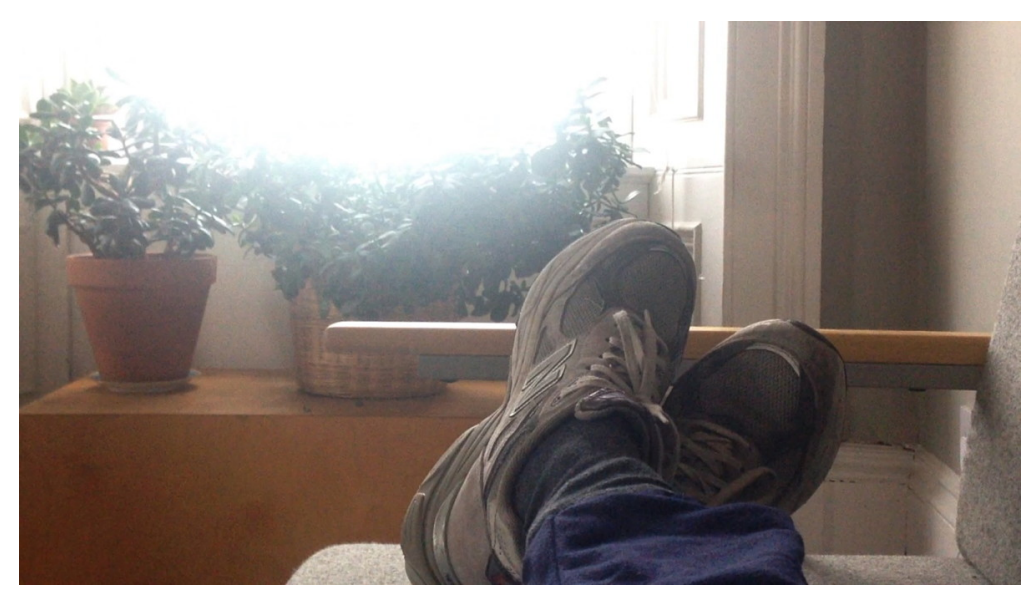

Abb. 1. Film-Still, BRIDGIT, Regie: Charlotte Prodger (Hollybush Gardens, Charlotte Prodger, 2016).

\section{PRIVATES KINO}

Die Eröffnungsszene von BRIDGIT zeigt aus einer subjektiven Perspektive die hochgelegten und übereinandergeschlagenen Beine und die sorgfältig gebundenen Sneakers von Charlotte Prodger (s. Abb. 1). Sie wurden mit dem iPhone gegen das aus dem Fenster ins Zimmer strömende Licht aufgenommen, während im Hintergrund das OnlineRadio NTS mit seinem charakteristischen Live-Musik-Programm lief. Man sieht die Grünpflanzen vor dem Fenster, die Szene wirkt privat und durch den rhythmischen, an ein Ritual erinnernden elektronischen Sound gleichzeitig fremd. Die Kamera ist statisch und schwankt zugleich leicht auf und ab, so dass das Bild dem Rhythmus der Musik $\mathrm{zu}$ antworten scheint. Es ist die atmende Bewegung des Körpers, der das iPhone hält. Dann hören wir über das Bild, den Sound und die Live-Stimme aus NTS Prodgers Stimme aus dem Off:

A group of people are focusing very closely on you. They might know details of you and also the macro.

Dann nach einer kurzen Pause, in der nur die Live-Musik aus NTS zu hören ist:

It's all women. They are totally in control of you. There are three main people in charge of you and an outer layer of others, each 
with their specific roles. And they all are focusing very intensely on you.

Wieder eine Pause, wieder das Live Event aus NTS und dann Prodgers ruhige Stimme:

You are at the centre of the whole thing. It's all about you. Every part of you. But you are not there!

Es folgt ein Schnitt und der Blick auf einen bewaldeten Hang im Nebel in einer Totalen, wir hören Vogelpfeifen und Waldgeräusche. Man fühlt sich immer noch als Zeugin eines Rituals, vielleicht einer Performance in einem subkulturellen Event und erfährt erst später, dass es sich bei der beschriebenen Szene um die Vorbereitung einer Narkose und einer nicht weiter spezifizierten Unterleibsoperation handelt. Die beschriebene Szene spielt sich in dem Übergangsraum (transition room) ab, der sich vor dem Operationsaal (operation theatre) und neben dem Aufwachraum (recovery room) befindet. Prodger liest den Text, der sich wie eine Tagebuchnotiz anhört, über ein Schwarzbild, das sich langsam in ein warmes Braun aufhellt. Die Menschen, die in der Eingangsszene benannt wurden, entpuppen sich als Narkoseärztin und Pflegefachkräfte. Prodger berichtet detailliert, was sie machen und zu ihr sagen. Sie versuchen, sie abzulenken und zu beruhigen, während sie ihr die nötigen Spritzen geben; dann lehnt sich die Anästhesistin über sie und sagt:

What you think about now is what you are going to dream about. So think about something nice.

Doch es bleibt nicht viel Zeit und Prodger versucht nun an ein Feld, ein Bild zu denken und es sich vorzustellen, aber es ist nicht das Richtige, sie kann das richtige Feld, die richtige Vorstellung nicht finden, so dass die Bilder sich zu bewegen und abzulösen beginnen:

Now this field, now that one like slides. I never settled on one and that slideshow, searching for the right field, was the last content before nothing.

Der Film, der sich aus Footage, manchmal nur aus kleinsten Schnipseln zusammensetzt, die Prodger über Jahre in ihrem iPhone-Video-Archiv sammelte, lässt sich als Aufführung einer ebensolchen Suche nach 
der $\gg$ richtigen Diashow « verstehen, die sich im Übergang und im Dazwischen von Wachsein und Wegsein ereignet. Mason Leaver-Yap beschreibt es als ein privates Kino, das sich direkt zum Körper hin öffnet, ohne jedoch auf die Präsenz des Körpers zu rekurrieren:

This is a private cinema that opens onto the artist's body in direct address. »It's all about you.« Yet the artist goes on to speak in voice-over not about the body's presence, whether hers or yours. Rather, she speaks about the inability to register presence at all - the total absence of self under the effect of anaesthetics. ${ }^{9}$

Die oft statischen Aufnahmen sind an einigen Stellen mit der akusmatischen Stimme von Prodger oder einer Freundin unterlegt, die tagebuchartige Texte und Notizen vorlesen, und mit sorgfältig ausgesuchtem, den Rhythmus des Bildes aufnehmenden Ton, dessen Quelle manchmal unsichtbar bleibt, manchmal aber auch im Bild zu sehen ist: Der Ton eines Schiffes, eines Zuges, einer Maschine; der Wind in einer Landschaft, der das kleine Mikro des iPhones zu überfordern scheint. Besonders schön ist die Szene, die eine schwarze Katze vor einer im Rhythmus der Jazzmusik zitternden, hellen Lampe zeigt. Das ganze Bild vibriert und scheint elektrisch aufgeladen, Träger einer virtuellen Kraft zu sein. Man kann nicht anders als an Bri, Breu oder Bridgit zu denken, die Göttin des Lichts und des Feuers. Für Leaver-Yap rekonfiguiert Prodger Subjektivität bis zu einem Punkt, an dem Beziehungen zwischen Körpern, Orten und Dingen nicht mehr als Nähe oder überhaupt als Beziehung zwischen Subjekten beschrieben werden können. Stattdessen handele es sich um eine transzendentale Idee von fluiden Beziehungen durch und über die Zeit hinweg, erscheinend als eine bewusste Distanzierung vom modernen Subjekt und dessen Überbetonung der Individualität und Rationalität der Einzelnen. ${ }^{10}$

\section{POLITIK UND ÄSTHETIK}

Der experimentelle Umgang mit dem iPhone als Kamera und Bildarchiv, der biografische Bezug, der Bezug zu Julian Cope, die Präsenz

9 Leaver-Yap, »Aesthetics and Anaesthetics in Charlotte Prodgers BRIDGIT «.

10 Ebd. 
der queeren Subkultur in Aberdeen und Aberdeenshire und das Interesse an der Formulierung einer fluiden und situierten Subjektivität knüpfen an queere Ästhetiken an und richten sich an eine queere Community. Tatsächlich bezieht sich der Film nicht nur auf eine soziale und politische Realität, die außerhalb der Kunstwelt liegt, er will auch verändernd auf diese einwirken. Prodger formuliert dies klar in dem 5-minütigen Video für die Tate Gallery, in dem sie ihre Arbeit vorstellt: Ihr seien die queeren Kämpfe und die Erinnerung daran wichtig und dies auch und gerade heute, wo es einen Zugriff auf queere Räume gäbe, der zum Teil auf die Kommerzialisierung von queeren Ästhetiken in der Mode, der Musik und der Kunstwelt zurückzuführen sei. Durch die Ablösung der ästhetischen Formen komme es zu einer Entleerung des politischen Inhaltes, der ursprünglich so wichtig gewesen sei. ${ }^{11}$

Damit positioniert sie sich in kritischer Distanz zu aktuellen Zelebrationen von Vielgeschlechtlichkeit, die wesentlich von der Aufmerksamkeitsökonomie der digitalen Medien geleitet sind und die Anpassung an die Mehrheitsgesellschaft, deren Beifall und Anerkennung suchen. Verglichen mit den neoliberal-digitalen Produktionsbedingungen der Popkultur eröffnet die Kunstwelt aktuell Möglichkeitsräume, in denen politische und medienästhetische Probleme erforscht werden können: in denen die Ästhetik an die Politik zurückgebunden und mit neuen ästhetischen Formen und Medien, wie im Falle von BRIDGIT mit dem iPhone, experimentiert werden kann. ${ }^{12}$

Diese Beobachtung bestätigend hat der Hamburger Filmwissenschaftler Daniel Kulle vor kurzem vorgeschlagen, von Filmen und Videos, die sich an den Rändern dieses Mainstreams bewegen, als »queere Experimentalfilme « zu sprechen. ${ }^{13}$ Kulle meint damit Filme,

11 »Charlotte Prodger | Turner Prize Winner 2018 | TateShots «, Tate Britain, YouTube, 17. September 2018, 5:17 min, hier 3:06-3:43 <https://www.youtube.com/watch? $\mathrm{v}=$ AsVWk5DlbCE $>$ [Zugriff: 3. Juni 2021].

12 Ich denke an die Documenta 14 unter der künstlerischen Leitung von Adam Szymczyk, die gleichzeitig in Kassel und Athen stattfand, oder an die Documenta 13 unter der Leitung von Carolyn Christov-Bakargiev zum Thema $\gg$ Collapse and Recovery « und ebenso an die 58. Biennale Venedig 2019, an der u. a. Prodgers SaF05 gezeigt wurde und der afroamerikanische Videokünstler Arthur Jafa mit der EinkanalVideoprojektion The White Album, in der er Whiteness und die zugehörige Ideologie der White Supremacy kritisch analysiert, den Goldenen Löwen für den besten Teilnehmer der internationalen Ausstellung »Living in Interesting Times « gewann.

13 Daniel Kulle, »Innovation an den Rändern des Queer Cinema. Ästhetische Strategien des Queeren Experimentalfilms «, in Queer Cinema, hg. v. Dagmar Brunow und Simon Dickel (Mainz: Ventil, 2018), S. 226-44, hier S. 226. 
die sich $\gg$ in ästhetischer und politischer Opposition zum heteronormativen Mainstream wie auch zu assimilationistischen Strömungen der LGBT-Bewegung « befinden; ${ }^{14}$ die also die oben zitierte Kritik von Prodger teilen. Der queere Experimentalfilm ist nach Kulle durch seine »systematische Zwitterstellung zwischen Kunst und Kino « charakterisiert. Er stellt ein $\gg$ hochgradig volatiles Feld $>$ dazwischen $<$ dar, zwischen Kunst und Film, zwischen der Filmwelt der Festivals und der von YouTube und Vimeo ${ }^{15}$

In der Tat wies Prodger in einem Interview anlässlich der Nominierung für den Film London Jarman Award 2017 darauf hin, dass das Kino die ideale mediale Umgebung für BRIDGIT sei. ${ }^{16}$ Gezeigt wurde der Film bisher jedoch nicht im Kino, sondern als Single-Screen-Video in simulierten Kinosituationen, in Projektionsräumen in Galerien und Ausstellungsräumen - etwa in der renommierten, 2005 von Lisa Panting und Malin Ståhl gegründeten Galerie Hollybush Gardens, die sich als Experimentierfeld für visuelles Denken versteht und die, neben Prodger, auch Andrea Büttner vertritt, die 2017 für den Turner Prize nominiert war, und Lubaina Himid, die den Turner Prize 2017 erhalten hat.

Alternativ kann BRIDGIT bei der britischen Kunst- und Vertriebsagentur LUX angefordert werden und, je nach Bedarf und Preis, für Forschungszwecke gesichtet, in Lernumgebungen eingesetzt oder öffentlich gezeigt werden. LUX ist eine 2002 gegründete, gemeinnützige und nicht nichtkommerzielle Organisation, welche sich in der Nachfolge der London Film-Makers' Co-op (LFMC) als Teil der $\gg$ Counter Culture « versteht, die bis in das London der 1960er Jahre zurückreicht. Die London Film-Makers' Co-op war von The Film-Makers' Cooperative inspiriert, die 1962 u. a. von Jonas Mekas, Shirley Clarke und weiteren zwanzig Filmkunstschaffenden in New York gegründet wurde und der Verbreitung avandgardistischer Filmkunst gewidmet ist. Die London Film-Makers' Co-op wurde in den 1970er und 1980er Jahren zu einem wichtigen und produktiven Ort für experimentelle

\footnotetext{
14 Ebd., S. 227.

15 Ebd.

16 >CHARLOTTE PRODGER - shortlisted artist profile - Film London Jarman Award $2017 \ll$, Film London, Vimeo, 23. August 2017 <https://vimeo.com/230803422> [Zugriff: 3. Juni 2021].
} 
visuelle Kunst. 1976 formierte sich die Gruppe London Video Arts, um die Produktion und Distribution der neu entstehenden Videokunst zu fördern. 1994 schließlich gründete sich London Electronic Art und 1997 zogen alle drei selbstverwalteten Organisationen in das Lux Centre, in dem eine Galerie, ein Kino, ein Archiv und Produktionsräume zur Verfügung standen. An diese Bewegungen schloss 2002 LUX mit dem Ziel an, die Sammlungs- und Distributionsaktivitäten fortzusetzen. ${ }^{17}$ Dass Charlotte Prodger ihre Filme über LUX vertreibt, zeigt, wie konsequent sie die Politik der Unabhängigkeit versteht und auch im Hinblick auf die Distribution ihrer Arbeiten betreibt.

\section{DER KÖRPER ALS VIRTUELLER ORT VON EMPFINDUNGEN}

Daniel Kulle kommt im Fazit seines Textes zu dem Ergebnis, dass der »queere Experimentalfilm « kein Genre, sondern eine »lose Gruppe von Filmen umfasst, die sich der Kategorisierung weitgehend entzieht $\ll .{ }^{18}$ Anstelle einer Kategorisierung macht er drei »ästhetische Reibungsflächen « aus, an denen, wie er schreibt »diese Filme mit dem Mainstream der LGBT-Festivals kollidieren können $\ll .{ }^{19} \mathrm{Er}$ charakterisiert diese Reibungsflächen als »subversive ästhetische Strategien, die sich im Sinne einer Mikropolitik der Macht gegen hegemoniale ästhetische wie politische Normen wenden $\ll{ }^{20}$ Diese von Kulle identifizierten ästhetischen Strategien lassen sich auch in BRIDGIT ausmachen.

Die erste dieser Strategien bezieht sich auf die Herstellung von Evidenz und Sichtbarkeit. Sie richtet sich gegen die Marginalisierung, Verdrängung und Verleugnung von queeren Körpern, Lebensweisen und Begehren. Es geht dabei zentral um die $\gg$ Geschichtlichkeit des Körpers und die Spuren, die Zeit und Wandel an ihm hinterlassen,

17 Eine sehr schöne Einführung in die Politik der Initiative gibt die Autorin, Kunstkritikerin und ehemalige Direktorin von LUX Schottland, Mason LeaverYap, in einem Vortrag im Rahmen der Konferenz »Archeology \& Exorcisms: Moving Image and the Archive $\ll$, Art \& Education, Online-Video, Dezember 2017 <https://www.artandeducation.net/classroom/video/168604/mason-leaveryap-archaeology-exorcisms-moving-image-and-the-archive> [Zugriff: 3. Juni 2021].

18 Kulle, »Innovation an den Rändern des Queer Cinema «, S. 240.

19 Ebd.

20 Ebd. 
die Formbarkeit des Körpers, um Biopolitik und Gouvernementalität $\ll{ }^{21}$ Es geht darum, den Körper in seiner $\gg$ Vielseitigkeit und im Spannungsfeld zwischen erotischer Anziehung und semiotischer Aufladung « zu zeigen. ${ }^{22}$ Kulle zählt dazu die postpornografische Darstellung von Genitalien in all ihrer Diversität und Bedeutungsvielfalt ebenso wie das von Jack Halberstam als Alternative zur figurativen Sichtbarkeit vorgeschlagene Konzept der »queer abstraction « oder das von Renate Lorenz formulierte Konzept des $\gg$ abstract drag $\ll .{ }^{23}$ Dazu gehört auch die »exzessive Ästhetik des Camp«, mit der »lustvolle Traumwelten aufgebaut werden, in denen die Möglichkeit eines Andersseins an sich im Mittelpunkt steht $\ll{ }^{24}$

Auch in BRIDGIT ist die Frage nach dem Körper zentral. Der Körper erscheint hier jedoch weder in abstraction noch figurativ und auch nicht als Träger einer Utopie, sondern in prozessualer Verschränkung mit Technischem, Medialem und als eine subjektivierte relationale Raumzeitlichkeit. Man sieht den Körper nicht, sondern man empfindet ihn: Durch die Stimme(n) und die Spuren des Atmens in der Bewegung der Bilder, durch die erzählten Geschichten, durch die mit dem iPhone aufgenommenen Landschaftsbilder, durch den intensiven und sorgfältig ausgesuchten Sound, durch den Rhythmus, der den Film durchzieht. BRIDGIT adressiert den Körper nicht als eine materielle Substanz, sondern als virtuellen Ort von Empfindungen und Intensitäten in einem Beziehungsgefüge mit technischen Objekten, das man mit dem französischen Philosophen Gilbert Simondon als $\gg$ transindividuelles « Beziehungsgefüge verstehen kann. ${ }^{25}$ Dabei ist mit virtuell hier nicht das digitale Virtuelle gemeint, sondern es ist

21 Ebd., S. 229.

22 Ebd.

23 Ebd., S. 232.

24 Ebd., S. 234.

25 Für Simondon ist die »transindividuelle Relation « an ein im emphatischen Sinn »technisches Denken« gebunden. Dieses »technische Denken« ist die Voraussetzung dafür, dass sich über der »Gemeinschaft der Arbeit «, in der Technik allein als Instrument in einer durch Herrschaft geprägten Beziehung zur Natur gedacht werden kann, ein »geistiges und praktisches Universum der Technizität [errichtet, d. V.in], in dem die Menschen vermittels dessen kommunizieren, was sie erfinden «. In dieser Beziehung wird das technische Objekt als »erfundenes, gedachtes und gewolltes, als Objekt [erfasst, d. V.in] dessen sich ein menschliches Wesen angenommen hat «; siehe Gilbert Simondon, Die Existenzweise technischer Objekte (Zürich: Diaphanes, 2012), S. 228. 
im Sinne der transzendentalphilosophischen Modallogik zu verstehen, die mit einer langen Tradition in die mittelalterliche Philosophie zurückreicht und auf die sich die Prozessphilosophie von Simondon ebenso bezieht, wie jene von Peirce und Deleuze. Das Virtuelle meint in diesem Sinn eine Kraft zur Veränderung der Aktualität und das meint eine Kraft zur Veränderung der Gegenwart. Als eine Kraft des Werdens ist das Virtuelle, wie Gilbert Simondon ausführt, zugleich in der Zukunft und in der Vergangenheit und vermag damit die Zukunft als Potentialität in die Gegenwart zurücklaufen zu lassen. ${ }^{26}$

Der Film gibt viel zu sehen und es geht zentral um den Körper und um Materialität. Materie wird jedoch nicht als eine Substanz oder Matrix adressiert, und der Körper auch nicht als Organismus mit definierten Grenzen zu einer Um- oder Außenwelt gedacht. Statt eine Evidenz zu vermitteln wird vielmehr die Vorstellung, man könne sich die richtige Vorstellung machen, oder sich auf eine richtige Vorstellung berufen, selbst fraglich. Der Körper verliert seine Grenzen und die Kontrolle entgleitet ebenso wie die Vorstellung, der Körper sei ein Eigentum und das Denken ein Kontrollorgan. Der Körper erscheint als Zentrum von Empfindungen und Intensitäten in einem transindividuellen Beziehungsgefüge.

\section{LEDA UND DER SCHWAN, QUEER-LESBISCHES BEGEHREN}

Die zweite ästhetische Strategie in Filmen, die als >queere Experimentalfilme« gelten können, besteht, so Kulle, darin, eine eigene

26 Ebd., S. 132. Simondon führt den Begriff des Virtuellen im Kontext der Unterscheidung von Grund und Form anstelle von Stoff, bzw. Materie und Form als Bedingung für die Möglichkeit von technischen Erfindungen ein. Während er die Gestalttheorie dafür kritisiert, dass sie den Grund nicht radikal genug gedacht habe und nach wie vor der Form die gestaltende Kraft zuspricht, wendet er dieses Kräfteverhältnis unter Rekurs auf die transzendentalphilosophische Modallogik, die mit einer langen Tradition auf Duns Scotus zurückreicht, um. Demnach ist $\gg$ der Grund das System der Virtualitäten, der Potentiale, der Kräfte, die sich langsam ihren Weg bahnen, während die Formen das System der Aktualität sind «; siehe ebd., S. 54. In dieser Tradition ist das Virtuelle nicht weniger real als das Aktuelle. Die Differenz zwischen Virtuellem und Aktuellem setzt ein Prozessdenken voraus. Das meint: Das Aktuelle ist eine Realität, die durch den Prozess der Aktualisierung gegangen ist. Das Virtuelle ist aktuell geworden und nicht länger virtuell. Vgl. Astrid Deuber-Mankowsky, »>Für eine Maschine gibt es kein echtes Virtuelles<: Zur Kritik des Smartness Mandate mit Felwin Sarrs Afrotopia und Gilbert Simondons Philosophie der Technik«, in Digital/Rational, hg. v. Dieter Mersch und Katerina Krtilova (= Internationales Jahrbuch für Medienphilosophie, 6, (2020)), S. 131-45. 
»queere Stimme« bzw. Erzählposition zu finden. Dabei meint Stimme $\gg$ sowohl eine queere Erzählposition [...], von der aus Sinn in die heteronormative Welt getragen wird, aber auch ganz explizit die körperliche oder akusmatische Stimme $\ll .{ }^{27}$ Die akusmatische Stimme werde, so führt Kulle unter Berufung auf die Medienwissenschaftlerin Robin Curtis aus, zu einem »autobiografischen Marker $\ll .{ }^{28}$ Während das Voiceover eine persönliche Geschichte erzählt, stellt es sich einer davon zunächst unabhängigen Bilder- und Soundwelt entgegen. Diese Bilder können Stadt-, Kultur- oder Naturlandschaften sein. Es können auch Körper sein, die zu der Stimme in einem Kontrast stehen. Kulle nennt als Beispiel dafür El Abuelo von Dino Dinco, der eine »sehnsüchtige und zärtliche Stimme $[\ldots]$ einem gangtätowierten, muskulösen Körper « entgegensetzt. ${ }^{29}$ Dieses Verfahren ist nicht nur für eine lange lesbische und schwule Filmgeschichte charakteristisch, sondern, wie Kulle unterstreicht, zugleich eine seit den 1960er Jahren »klassisch gewordene ästhetische Strategie jeglicher gegenkulturellen Video- und Filmproduktion von Minoritäten $\ll .{ }^{30}$ Die Gegenüberstellung von Off-Stimme und Bilder- und Soundwelten ermögliche die Formulierung einer minoritären Position und die gleichzeitige Unterwanderung der etablierten Repräsentationen, denen sie sich gegenübersieht. Durch den Kontrast zwischen Bildwelt und akusmatischer Stimme werde, so Kulle, der $\gg$ vermeintlich unproblematische Individuationsprozess, den die Stimme performativ behauptet, jedoch gleichzeitig widerrufen $\ll .{ }^{31}$ Das queere Selbst sei, so Kulle, damit notwendiger Weise ein $\gg$ ironisches und metasubjektives: Indem das Selbst sich aus der Reibung an den Bildwelten heraus entwickelt, die Differenz zu diesen aber niemals überwinden kann, bleibt es als Konstrukt ohne Fundament, ein reines Provisorium $\ll .{ }^{32}$

Dass auch Prodger in BRIDGIT mit akusmatischer Stimme und Landschaft arbeitet, ist bereits deutlich geworden. Und mehr noch: Auch die Möglichkeiten der Generierung einer queeren Erzählpositi-

27 Kulle, »Innovation an den Rändern des Queer Cinema «, S. 234.

28 Ebd.

29 El Abuelo, Regie: Dino Dinco (Dino Dinco, 2004); Kulle, »Innovation an den Rändern des Queer Cinema «, S. 235.

30 Ebd.

31 Ebd.

32 Ebd. 
on im Kontext der digitalen Remix Culture und des Found Footage, auf die Kulle hinweist, lassen sich wiederfinden. So setzt sich der Film aus Videosequenzen zusammen, die Prodger mit ihrem iPhone filmte und digital archivierte. Es handelt sich bei diesem Footage allerdings nicht, wie in den Beispielen von Kulle, um popkulturelle Bilderwelten, sondern um gefundene Bilder aus dem eigenen, persönlichen Archiv, die in der Montage zu einem neuen Ausdrucksgefüge finden. Unter Bezugnahme auf Filme von Barbara Hammer, wie z. B. Nitrate Kisses oder Tender Fictions, ${ }^{33}$ bringt Kulle einen weiteren auch für die Analyse von BRIDGIT wichtigen Punkt ins Spiel: So weist er darauf hin, dass Voiceover und Remix insbesondere in der Reflexion über Formen queerer Kollektivität zum $\gg$ Grundinventar des queeren Experimentalfilmes gehören $\ll .{ }^{34}$ Auch dieses Anliegen, neue Ausdrucksformen queerer Kollektivität zu kreieren, finden wir in BRIDGIT.

Ich werde im Folgenden entlang einer besonders beeindruckenden Einstellung, in der akusmatische Stimme(n), queere Kollektivität, biographische Erfahrungen und Landschaftsbild und Sexualität zusammenkommen, darzustellen versuchen, in welcher Weise BRIDGIT an die von Kulle vorgestellte ästhetische Strategie einer eigenen Stimme anknüpft und wo und wie der Film diese ästhetisch und politisch überschreitet.

Die Sequenz dauert zweieinhalb Minuten und befindet sich genau in der Mitte des Films. Es ist eine statische Einstellung, mit dem bereits vertrauten, kaum wahrnehmbaren, leichten Schwanken, welches den Atem und durch diesen die Anwesenheit des Körpers anzeigt, mit dem die Kamera verbunden ist. Auf dem Bild sind im Vordergrund bemooste Steine zu sehen, zwischen denen das Wasser ins Meer zurückfließt (s. Abb. 2 und Abb. 3). Es ist offensichtlich Ebbe. Am Rand des Strandes, bevor das offene Wasser beginnt, steht eine Gruppe von weißen Schwänen, die ihre langen Hälse elegant zum Trinken winden und beugen, ihr Federkleid putzen, sich am Ort bewegen. Fast ununterscheidbar von den Steinen stehen drei Enten mit eingezogenen Köpfen. Der Wind geht und wir hören ihn und die Wellen und se-

33 Nitrate Kisses, Regie: Barbara Hammer (Barbara Hammer, 1992); Tender Fictions, Regie: Barbara Hammer (Barbara Hammer, 1996).

34 Kulle, »Innovation an den Rändern des Queer Cinema «, S. 236. 


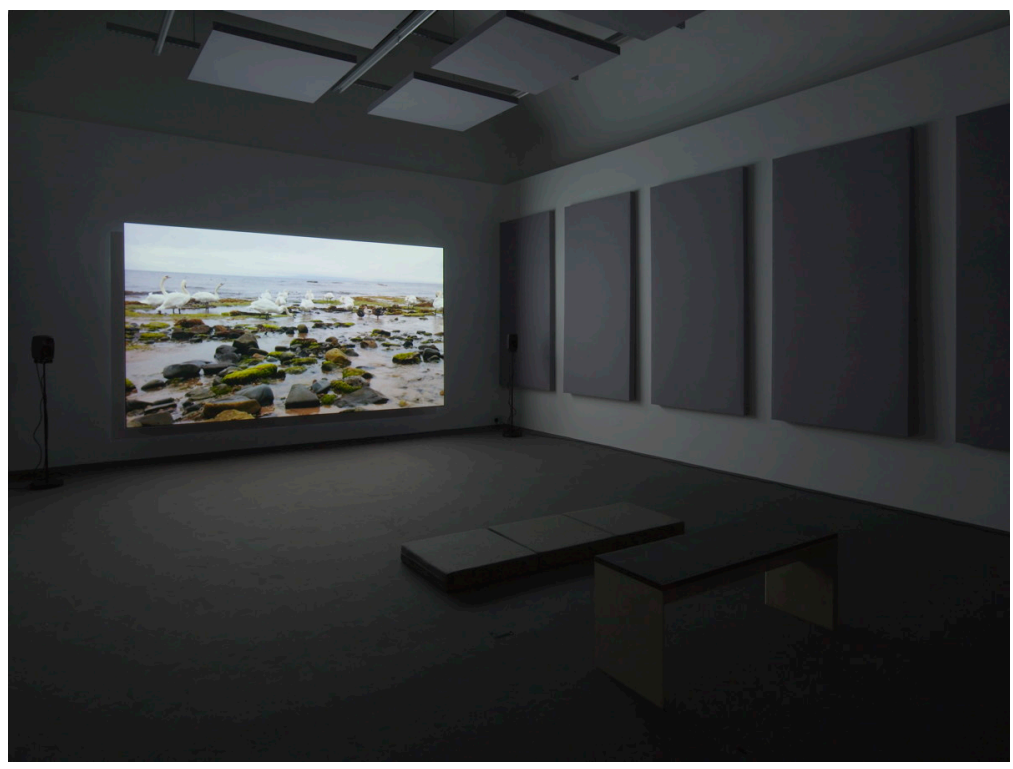

Abb. 2. Installation von BRIDGIT (Hollybush Gardens, London, 2016), Foto: Andy Keate.

hen am Horizont die Wolken sich bewegen. Manchmal fliegen Vögel durchs Bild.

Derweil liest die bereits bekannte Stimme, die nicht jene von Prodger ist, folgende Tagebuchnotizen vor:

November 14th: bought two t-shirts, a pair of jogging pants and some socks at jd sports. The check-out girl asked if it is my son I am buying for. I said, no it's me. She didn't say much after that.

January 28th: I am on a shift at the bar where I worked as a DJ. I put on a long record and went to the toilet [...]. One girl sees me in the queue and shouts: there is a boy in the girl's toilets.

March 23rd: Helen, in the bed next to me asks me if that was my daughter who visited me last night. I said no, actually it is my girlfriend. She raised her hands: Don't have a problem with that. My son is gay.

Es folgen weitere Einträge, die von der Unkenntlichmachung queeren Begehrens und lesbischer Lebensformen in einer heterosexuellen Umgebung handeln. Die Einstellung endet mit den folgenden Sätzen: 


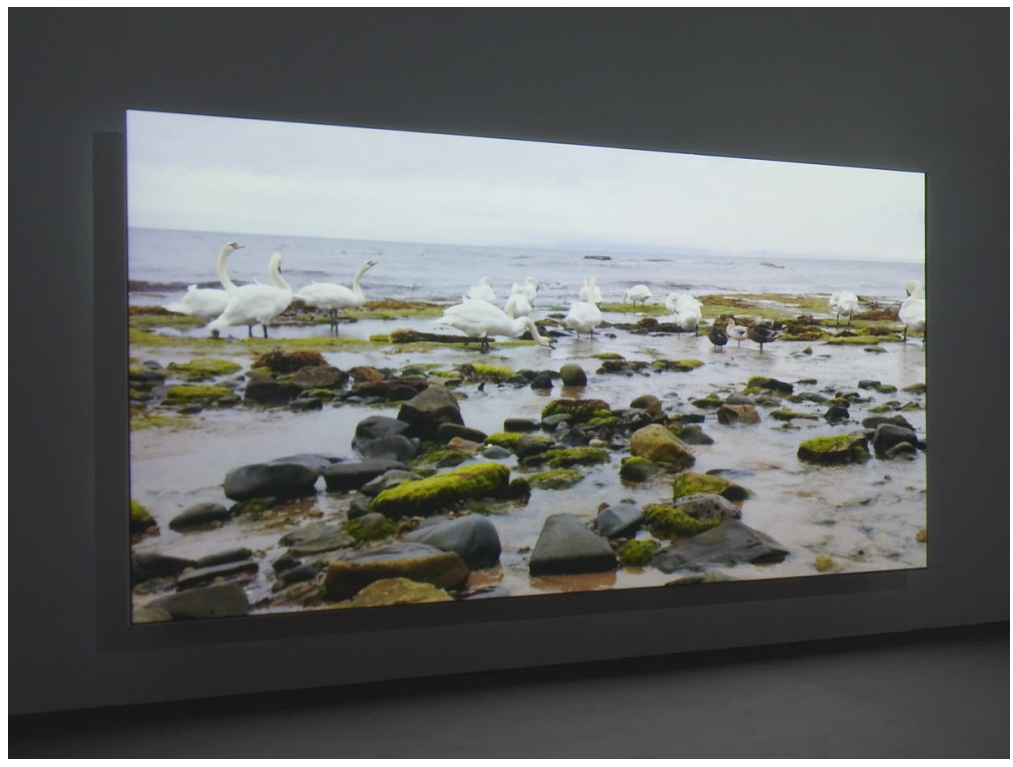

Abb. 3. Installation von BRIDGIT (Hollybush Gardens, London, 2016), Foto: Andy Keate.

I told Isabelle. She said that usually her and Ellen get: are you twins? And once Ellen got: is that your son? I told Irene. Wee has variously been her mother and her brother.

Das lesbische Begehren wird in diesen Aussagen in einer heteronormativen Umgebung mit der Beziehung von Zwillingen, von Mutter und Sohn und von Mutter und Tochter assoziiert. Statt dass das lesbische Begehren in dieser Operation der Ersetzung jedoch verschwindet, um unsichtbar und non intelligible zu werden, kommt es durch die Montage von Stimme, Erzählposition und zitierten Tagebucheinträgen zu einer Überlappung von erotischer und verwandtschaftlicher Beziehung. Diese führt zu einer Erschütterung der symbolischen Position des Inzesttabus, das, wie Judith Butler in Antigones Verlangen. Verwandtschaft zwischen Leben und Tod gegen Lacan und gegen LeviStrauss argumentierte, seinerseits auf der vorgängigen Verdrängung und Tabuisierung der gleichgeschlechtlichen Liebe beruht. ${ }^{35}$ In der

35 Judith Butler, Antigones Verlangen: Verwandtschaft zwischen Leben und Tod, übers. v. Reiner Ansén (Frankfurt a. M.: Suhrkamp, 2001), S. 40. In Psyche der Macht 
beschriebenen Szene kehrt sich nun die Tabuisierung des lesbischen Begehrens auf einmal gegen das Tabu des Inzests selbst. Es widersetzt sich dem Verschwinden und stellt damit durch die Überlappung von Verwandtschafts- und von sexueller Beziehung zugleich das symbolische Gesetz des Inzesttabus in Frage. In der Szene wird nichts weniger als der Rahmen selbst sichtbar, der die Prekarität queerer Körper und queerer Sexualität hervorbringt und reproduziert.

Gleichzeitig scheint in der Szene des sich konstant in reflektierter Bewegung befindenden Wassers, der Wolken und der Schwäne das virtuelle Bild eines kraftvollen queeren sexuellen Begehrens auf. Selbst ohne die Anwesenheit einer Leda-Figur erinnern die Schwäne an kunstgeschichtliche Darstellungen der sexuellen Begegnung von Leda mit einem Schwan. In der schriftlichen Überlieferung verliebte sich Zeus in Leda und näherte sich ihr eines Nachts in Gestalt eines Schwans. Leda hatte in der gleichen Nacht Sex mit ihrem Gemahl, dem spartanischen König Tynadereos, wurde schwanger und gebar zwei Eier mit zwei Zwillingspaaren: Die unsterblichen Zwillinge Helena und Pollus und die sterblichen Zwillinge Klytaimnestra und Kastor. Während die Geschichte von Zeus und Leda in der schriftlichen Überlieferung als einer der vielen sexuellen Übergriffe von Zeus erzählt wird, löste sich demgegenüber die bildliche Darstellung der sexuellen Begegnung von Schwan und Leda von Zeus ab. Die Begegnung von Leda - der Name heißt, übersetzt, nichts anderes als Frau - und dem Schwan, wurde bereits in der Antike bildlich dargestellt und entwickelte sich in der Renaissance-Malerei zu einem beliebten erotischen Motiv. Ausschlaggebend für diese bildlichen Darstellungen und ebenso für die Szene in BRIDGIT ist nun, dass Zeus in diesen Bildern nicht gezeigt wird; er ist, anders formuliert, nicht anwesend und kann entsprechend weggedacht werden. Anwesend sind Leda, der Schwan, manchmal Cupido, oder die von Leda gelegten Eier, manchmal Gespielinnen von Leda oder ihre Mägde. Ohne Zeus jedoch wandelt sich

unterscheidet Butler zwischen einer $\gg$ heterosexuellen Melancholie « und einer $\gg$ homosexuellen Melancholie $\ll$, um die jeweilig unterschiedliche Wirkung und Dynamik der Tabuisierung der Homosexualität zu erklären, die, wie sie überzeugend darstellte, sowohl dem Ödipuskomplex als auch dem Inzestverbot vorausgeht, und damit die heteronormative Verfasstheit des Verwandtschaftssystems stützt; siehe Judith Butler, Psyche der Macht. Das Subjekt der Unterwerfung, übers. v. Reiner Ansén (Frankfurt a. M.: Suhrkamp 1997), S. 135. 


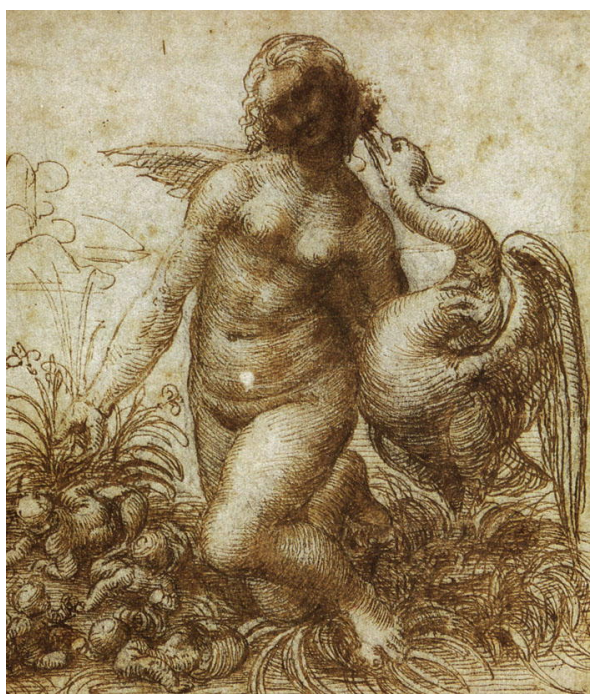

Abb. 4. Leonardo da Vinci, Leda und der Schwan (ca. 1508), Zeichnung auf Papier, $16 \times 13,9 \mathrm{~cm}$ <https://commons.wikimedia.org/wiki/File: Leonardo_da_vinci,_Leda_and_the_Swan_study.jpg > [Zugriff: 3. Juni 2021].

die Szene von Leda und dem Schwan in eine beglückende sexuelle Begegnung, die als eine nicht-heterosexuelle Szene auslegbar ist. Diese bildlichen Darstellungen wurden aus feministischer Perspektive als Verharmlosung von sexueller Gewalt gegenüber Frauen und entsprechend als Männerphantasien interpretiert. Sie können jedoch, wenn man nicht sieht, was auch nicht da ist - eben Zeus und die von ihm repräsentierte heteronormative Markierung der Sexualität - auch als nicht-heterosexuelle, queer-sexuelle Szenen wahrgenommen werden. Überliefert sind, um nur einige Beispiele zu nennen, die Studien von Leonardo da Vinci und eine Skizze, auf der auch die Eier zu sehen sind, die Leda gebar und auf der Leda den Schwan zärtlich umarmt (s. Abb. 4). Oder das Ölgemälde von Correggio, das in der Gemäldegalerie in Berlin hängt und die Szenen in einem Bild verbindet, in denen sich Leda und der Schwan treffen, sich sexuell vereinigen und danach voneinander trennen (s. Abb. 5). Auf dem Bild des RokokoMalers François Boucher, der ein Vertrauter und Freund von Madame de Pompadour war, liegen zwei Frauen beieinander und heißen den Schwan mit offensichtlicher Freude willkommen (s. Abb. 6). In BRID- 


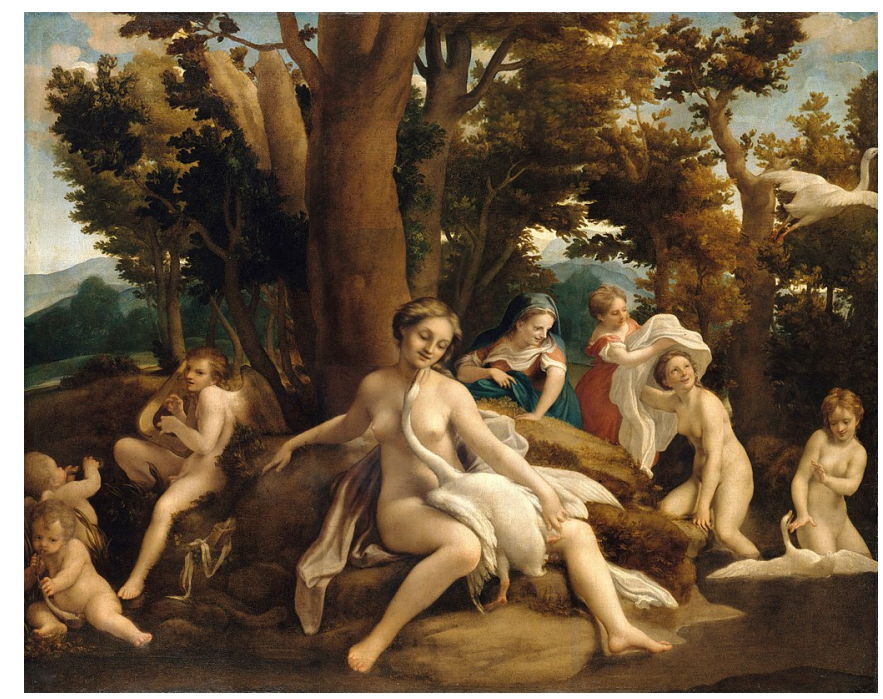

Abb. 5. Antonio da Correggio, Leda mit dem Schwan (ca. 1532), Ölgemälde, 156,2 $\times 217,5 \mathrm{~cm}<\mathrm{https}$ ///commons.wikimedia.org/wiki/File:

1532_Correggio_Leda_mit_dem_Schwan_anagoria.JPG> [Zugriff: 3. Juni 2021].

GIT sieht man nur Steine, die Schwäne und die drei Enten. Sie werden jedoch ebenso wie das Wasser, die Wolken, die Landschaft zu Verbündeten eines queer-lesbischen Begehrens, das die Szene über die Bewegung des Atmens mit dem Körper von Charlotte Prodger verbindet, die das iPhone hält.

Prodger trägt die alltäglichen Situationen des Ausschlusses und der Pervertierung queer-lesbischen Begehrens in ihrem Film in den Raum der Ästhetik, wo sie zum Ausgangspunkt für die Erschaffung von etwas Neuem werden. Wir können die Einstellung mit Deleuze als einen »bloc de sensations «, einen Empfindungsblock, auslegen. In einem Empfindungsblock wird, wie Deleuze ausführt, flüchtigen, gelebten und vergänglichen Empfindungen ein Halt im Sinne eines Da-Seins gegeben. ${ }^{36}$ Kunst wird damit nicht nur zum ästhetischen Ausdrucksmedium von Empfindungen, sondern Kunst bringt zugleich die Virtualität zum Ausdruck, die diesen Empfindungen als Intensitäten zukommt. Diese Virtualität ist hier die Kraft eines queer-lesbischen

36 Gilles Deleuze und Félix Guattari, Was ist Philosophie?, übers. v. Bernd Schwibs und Joseph Vogl (Frankfurt a. M.: Suhrkamp, 2000), S. 191. 


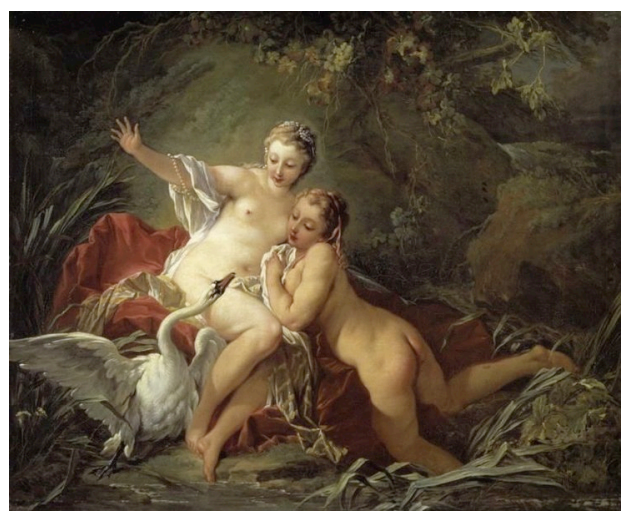

Abb. 6. François Boucher, Leda und der Schwan (1742), Ölgemälde, 59,5 $\times 74 \mathrm{~cm}<$ https://commons.wikimedia.org/wiki/File: Boucher_Leda_och_svanen.jpg $>$ [Zugriff: 3. Juni 2021].

Begehrens, das in der Szene aufscheint. ${ }^{37}$ Die Bilderwelten werden ebenso wie die in den Bildern zu sehenden Landschaften und Tiere im Sinne eines fluiden und transindividuellen Beziehungsgefüges zu Verbündeten in der Herausbildung einer starken, fluiden queeren Identität.

\section{DAS IPHONE ALS EIN MEDIUM QUEERER ÄSTHETIK}

Die dritte und letzte von Kulle analysierte ästhetische Strategie des queeren Experimentalfilmes trägt die Überschrift »Performanz des Selbst $\ll .{ }^{38} \mathrm{Zu}$ den Filmen, in denen es um die performative Aufführung eines queeren Selbst geht, zählt Kulle Filme, in denen Dragqueens und Dragkings im Zentrum stehen. Auch hier wird ein $\gg$ Queeres Selbst « mit »Ironisierung « und mit der Überschreibung von Natürlichem mit Künstlichem verbunden. Dazu kommt, dass diese Filme als Low-/No-Budget-Produktionen eine $\gg$ Do-it Yourself-Kultur « als Gegenmodell entwickelten und das meint, mit begrenzten Ressourcen und technischen Mitteln experimentierten.

37 Vgl. Astrid Deuber-Mankowsky, Queeres Post-Cinema. Yael Bartana, Su Friedrich, Todd Haynes, Sharon Hayes (Berlin: August Verlag, 2017), S. 68-71. 
An diesem Punkt könnte man Charlotte Prodgers Entscheidung, mit einem iPhone zu filmen, in der Tradition der queeren Experimentalfilme verorten. Prodger selbst betont, dass die Bedingungen der technischen Formate, mit denen sie arbeitet - seien dies 16-mm-Filme oder eben ein iPhone, ja selbst Drohnen-Kameras ${ }^{39}$ - eng mit dem autobiografischen Inhalt ihrer Filme verbunden sind. ${ }^{40}$ Allerdings bedeutet das nicht, dass sie in Kulles Sinn »>eine Ästhetik des Fragmentarischen, des nicht Abgeschlossenen, nicht Fertigen $<\ll$ sucht. $^{41}$ Im Gegenteil: Präzision und konsequentes Durchdenken spielen eine zentrale Rolle. So ist es kein Zufall, wenn wir an einer Stelle für einen Augenblick die helle Fingerkuppe von Prodger im Bild sehen, sondern es sind, ebenso wie das hauchzarte Schwanken bei statischen Weitwinkel-Aufnahmen, die über zwei Minuten dauern und ohne Stativ gefilmt wurden, bewusst gesetzte materielle Spuren des Körpers, die für Prodger auf das spezifische Verhältnis von Körper und Technik im Filmen mit einem iPhone verweisen. Tatsächlich vermitteln sich die technischen Bedingungen für Prodger auch im Fall der digitalen Technik über Empfindungen, das heißt über ästhetisch vermittelte materielle Spuren mit dem Körper. Dies wird etwa deutlich, wenn sie beschreibt, dass das iPhone im Prozess des Filmens sehr materiell, geradezu $\gg$ skulptural « geworden sei. ${ }^{42}$ Man könne, so führt sie aus, das iPhone mitten in der Aufnahme drehen, die Finger können im Bild sichtbar werden, man kann das Blut in den eigenen Fingern sehen, wenn man sie nah an die kleine Kamera hält; wenn man in der Landschaft filmt, führt der Wind das kleine Mikrofon an seine Grenzen, zerreißt es fast und wenn man eine statische Aufnahme macht, sieht man den Körper atmen. Der Köper und seine Systeme verschränken sich mit der Kamera. Es ist eine Art Symbiose und zugleich eine Art Verklammern. In diesem bedachten Umgang erscheint das iPhone im emphatischen Sinn als ein $\gg$ technisches Objekt $\ll$, von dem Simondon forderte, dass dieses als ein »erfundenes, gedachtes und gewolltes « zu behandeln sei. ${ }^{43}$ Nur so könne es zu einem Element in einem

\footnotetext{
39 Vgl. den Beitrag von Henriette Gunkel in diesem Band.

40 >CHARLOTTE PRODGER - shortlisted artist profile - Film London Jarman Award $2017 \ll, 6: 56 \mathrm{~min}$, hier 0:45.

41 Kulle, $\gg$ Innovation an den Rändern des Queer Cinema $\ll$, S. 239.

42 >Charlotte Prodger| Turner Prize Winner 2018| TateShots «, 5:17 min, hier 1:30.

43 Simondon, Existenzweise, S. 228. Vgl. auch Fußnoten 19 und 20.
} 
transindividuellen Beziehungssystem werden, das er $\gg$ Universum der Technik « nennt, »in dem die Menschen vermittels dessen kommunizieren, was sie erfinden $\ll .{ }^{44}$ In Prodgers Experimentieren erweitern sich, um es zusammenzufassen, mit dem Denken der Technik auch die Möglichkeiten und das Vermögen, den Körper zu denken. Eben darin unterscheidet sich BRIDGIT auch von anderen, immer zahlreicher werdenden Handy- oder iPhone-Filmen. ${ }^{45}$

Wenn Prodger das Filmen mit dem iPhone skulptural nennt, so kann das Video selbst als eine Skulptur beschrieben werden. Wir erinnern uns, dass Prodger dem Bewusstseinsverlust beim Fall in die Narkose mit dem Versuch begegnete, das richtige Vorstellungsbild zu finden, das sie während der Narkose begleiten sollte und dass dieser Versuch darin mündete, dass sich die Vorstellungsbilder wie in einer Diashow abzulösen begannen und sie gleichzeitig nicht die $\gg$ richtige Diashow « finden konnte. Das Aufwachen aus der Narkose ist wiederum mit einer Bewegung verbunden, die diesmal jedoch nicht horizontal, sondern vertikal ist: Wir sehen in einer statischen Aufnahme die Menhire eines keltischen Steinkreises in der Aberdeener Landschaft. In das Bild werden in der nächsten Einstellung weibliche Vornamen eingeblendet: »Margaret, Deborah, Eimear, Helen «. Im darauffolgenden, ebenfalls in gelben Buchstaben gesetzten Satz werden diese Namen als Punkte in einem sich bewegenden Gitter beschrieben. Und auf einmal wird klar: Bei diesem Gitter handelt es sich um die Darstellung des Bewegungsablaufes in einem Krankenhaus, in dem $\gg$ Margaret $\ll, ~ \gg$ Deborah $\ll, ~ \gg$ Eimear $\ll, ~ \gg$ Helen $\ll$ Namen von Frauen sind, die operiert werden. Sie kommen morgens in die Krankenstation, werden aufgenommen, werden im Lift in den tran-

44 Ebd.

45 Bei diesen mit einem Handy oder einem iPhone gedrehten Filmen handelt es sich zumeist um neue Formen von Dokumentarfilmen, vgl. Florian Krautkrämer, »Revolution Uploaded. Un/Sichtbares im Handy-Dokumentarfilm «, Zeitschrift für Medienwissenschaft, 6.2 (2014), S. 113-26, oder, wie bei den iPhone-Filmen von Steven Soderbergh, um Spielfilme, die sich an der Ästhetik des Kinofilms orientieren. Hier kommt das iPhone in erster Linie zum Einsatz, weil es günstiger ist und mehr Unabhängigkeit in der Realisierung von Filmprojekten ermöglicht. Dies gilt nicht nur für den iPhone-Film Unsane, Regie: Steven Soderbergh (Fingerpringt Releasing, Extension 765, New Regency Productions, 2018), sondern auch für die ebenfalls gänzlich per iPhone gefilmte Netflix-Serie High Flying Bird (Extension 765, Harper Road Films, 2019). 


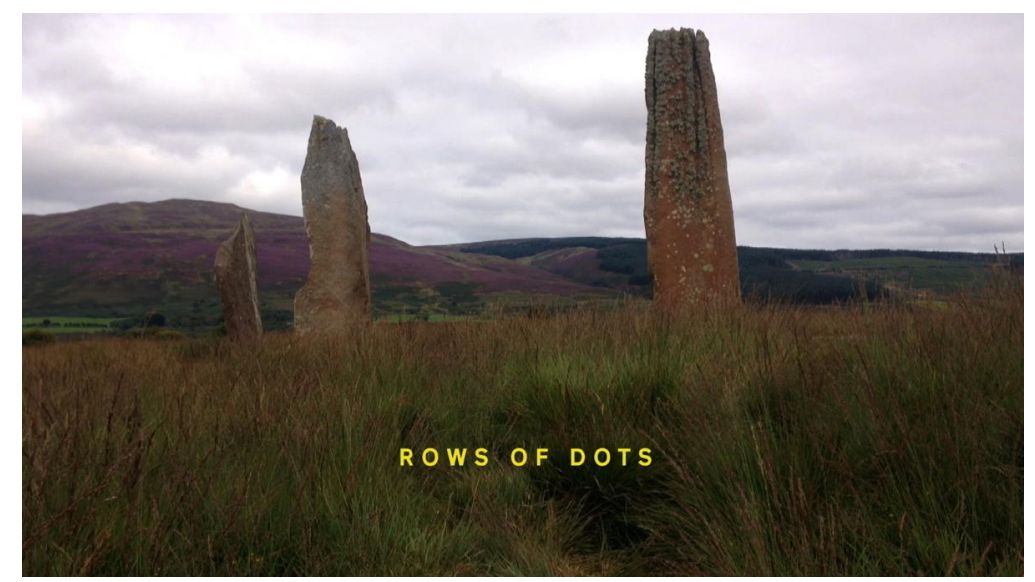

Abb. 7. Film-Still, BRIDGIT, Regie: Charlotte Prodger (Hollybush Gardens, Charlotte Prodger, 2016).

sition room hochgefahren, von dort in den Operationssaal gebracht, dann in den Aufwachraum, und mit dem Lift wieder in die Station zurückgefahren. Wir hören nur den Wind, der das Gras bewegt, und lesen die folgenden Sätze, die über die statische Einstellung auf die in der Landschaft stehenden Menhire eingeblendet werden:

Coming in at 7.30 / Waiting, then down the lift / into theatre / out to recovery, back to the ward, next ones come in, go down, go in, come out, go up.

Prodger vergleicht das Diagramm, das sich über die Bilder der Landschaft legt, mit den minimalistischen Bildern der lesbischen Malerin Agnes Martin. Der sich wiederholende Ablauf sei wie eine $\gg$ Agnes Martin with moving parts « - eine Reihe von Punkten, die sich in vertikalen Säulen, den Menhiren, bewegen (s. Abb. 7).

Im nächsten Schritt wird die Vorstellung des Steinkreises als einer Reihe von sich bewegenden Punkten noch einmal ausgeweitet. Er steht nun für einen »unendlichen Raum-Zeit-Rhythmus «, der, wie jetzt über den aus dem Gras zu wachsen scheinenden Steinsäulen zu lesen ist, da war, lang bevor $\gg$ ich «, das meint Charlotte Prodger, da war. Der unendliche Raum-Zeit-Rhythmus war, wie es weiter geschrieben steht, da, als sie diese Sätze schrieb und als sie den Film schnitt, und er ist da und geht weiter, während wir, das Publikum, den Film sehen. 
Prodger schreibt sich und uns auf diese Weise in eine Geschichte des Lebens ein, die sich nicht an der Ordnung des Stammbaums und der Verwandtschaft orientiert, sondern umfassender ist und die Geschichte der technischen Objekte ebenso umfasst wie jene der Lebewesen und der Landschaft.

Die nächste Einstellung beginnt mit einem Schwarzbild und wir hören Prodgers Stimme die verschiedenen Namen der trans* Medientheoretikerin, Soundtechnikerin, Performerin und Aktivistin $\gg$ Alluquére Rosanne $>$ Sandy $<$ Stone $\ll$ aufsagen, es klingt fast wie ein Reim oder ein Gedicht und erinnert an die vielen Namen von Bridgit. Damit wird die 1936 geborene, unter den Namen Sandy Stone bekannte Autorin einschlägiger cyberfeministischer Texte und des ebenso einschlägigen 1987 veröffentlichten The Empire Strikes Back: A Posttranssexuell Manifesto, in enge Verbindung mit der fluiden Identität der keltischen Gottheiten gebracht. Nicht nur das: Die trans* Frau Sandy Stone, aus deren Texten der 1990er Jahre in BRIDGIT im Folgenden zitiert wird, erscheint mehr noch als Vordenkerin eben dieses Konzeptes einer fluiden Identität und der Verschränkung von Technik, Körper, Zeit und Geschlecht und der Idee, dass eine entkörperte Subjektivität mit Queerness durchaus zusammengeht.

Der Bezug auf Sandy Stone ist in vielen Hinsichten eminent politisch. In einer der nächsten Einstellungen zitiert die Off-Stimme Prodgers das online veröffentliche Interview mit Sandy Stone über ihr Verhältnis und ihre Erfahrungen mit dem lesbian separatism in den 1970er Jahren. ${ }^{46}$ Sandy Stone arbeitete in jener Zeit als Tontechnikerin für den Frauen-Musik-Verlag Olivia Records. Olivia Records bekannte sich offiziell zu einem radikalen Feminismus und privat zu einem lesbischen Separatismus, heißt es im Interview. Anders als Vertreterinnen des radikalen Feminismus, wie Janice Raymond, die Sandy Stone unterstellten, keine »echte « Frau zu sein und das Label dafür kritisierten, mit einer trans* Frau zu arbeiten, hatten die lesbischen Separatistinnen kein Problem damit, die transsexuelle Sandy Stone in ihre lesbische Gemeinschaft zu integrieren. Im Gegenteil, sie fühlten

46 Zackary Drucker, »Sandy Stone on Living Among Lesbian Separatists as a Trans Woman in the 70s $\ll$, Vice, 19. Dezember $2018<$ <ttps://www.vice.com/ en_us/article/zmd5k5/sandy-stone-biography-transgender-history> [Zugriff: 3. Juni 2021]. 


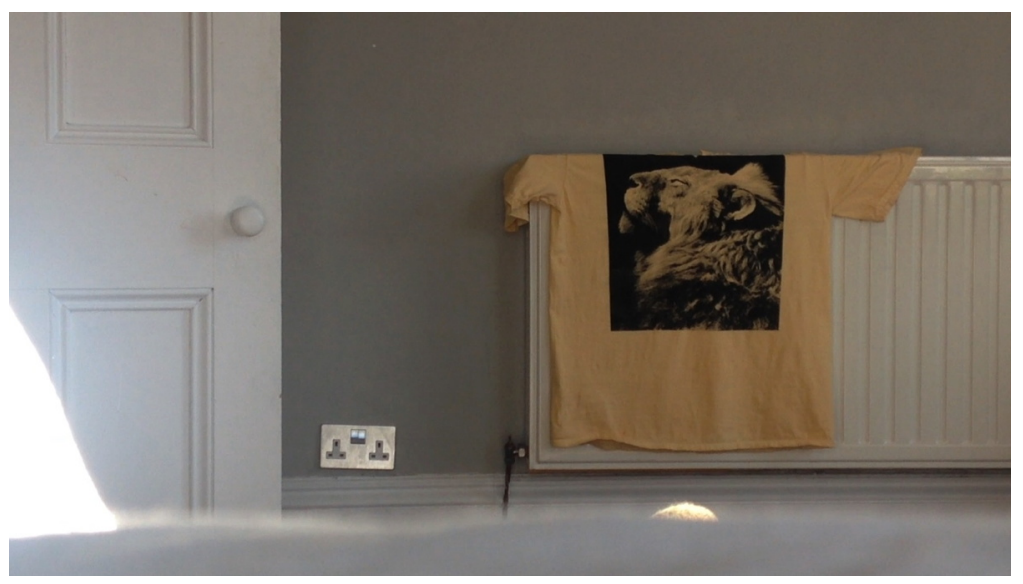

Abb. 8. Film-Still, BRIDGIT, Regie: Charlotte Prodger (Hollybush Gardens, Charlotte Prodger, 2016).

sich Sandy Stone in vieler Hinsicht näher als vielen cis* Frauen. Für Prodger stellten sie damit eine Form des lesbischen Separatismus vor, die relational und sich stetig verändernd ist.

Man muss diese Stellungnahme einerseits vor dem Hintergrund der aktuellen Kämpfe sehen, die in Großbritannien die Vertreterinnen der TERF (Trans-Exclusionary Radical Feminists) gegen die trans rights, die Rechte von trans* Menschen, führen, da sie eine Auslöschung von cis* durch trans* Frauen befürchten. Vor diesem Hintergrund erst wird deutlich, dass die prominente Bezugnahme auf Sandy Stone in BRIDGIT zugleich eine Distanzierung von der Politik der TERF bedeutet. Andererseits jedoch ist Prodgers Stellungname für einen lesbischen Separatismus, der eine fluide und relationale Identität affirmiert, auch eine Absage an eine Identitätspolitik, die Identität mit einem starken Selbst und einer partialen Gruppe verbindet. Dies zeigt sich deutlich in den letzten beiden Einstellungen von BRIDGIT. Die erste spielt Zuhause und zeigt ein über einer Heizung hängendes TShirt mit dem Kopf eines Löwen, der unschwer als jene queere Löwin zu erkennen ist, die in Prodgers nächstem, auf der Biennale von Venedig gezeigten Video SaFo5 die Hauptrolle spielen wird (s. Abb. 8). Es handelt sich um eine von jenen Löwinnen, die in Botswana im Okavangodelta von Überwachungskameras beobachtet wurden, die 


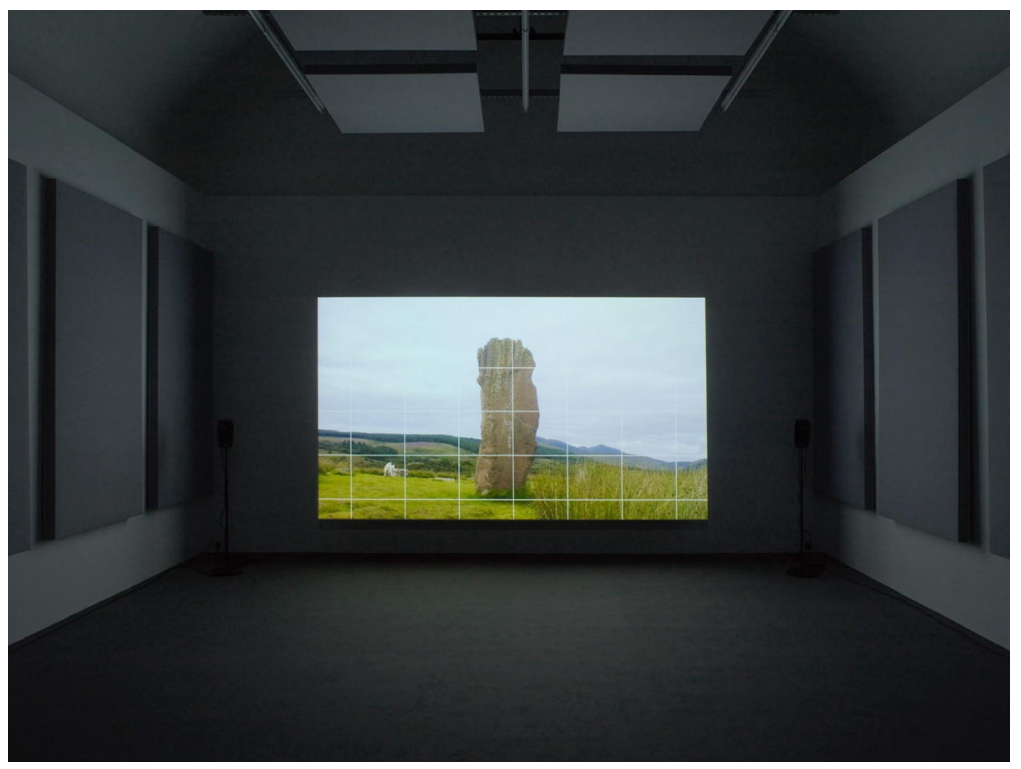

Abb. 9. Installation von BRIDGIT (Hollybush Gardens, London, 2016), Foto: Andy Keate.

sich wie Löwen verhalten, eine Mähne haben, alleine auf die Jagd gehen und aber eben doch Löwinnen sind. ${ }^{47}$ Die stolze, gegen den Himmel gerichtete Haltung des bemähnten Löwinnenkopfes begleitet uns in die nächste und letzte Szene, die eine Steinsäule in einer Landschaft zeigt, überblendet von einem weißen Gitter, das sich weitet und schließlich auflöst (s. Abb. 9). Es bleibt die Steinsäule aus der Jungsteinzeit, die ebenso an Bridgit erinnert, wie in ihrem oberen Ende an die stolze Mähne der queeren Löwin SaFo5.

Es gab eine solche queer lioness übrigens auch im Zoo von Oklahoma. Dieser Löwin wuchs im Alter von 18 Jahren auf einmal ein Bart. Das war genau zu der Zeit, als Prodger den Film fertigstellte. Die Löwin mit der Mähne hieß: Bridget. Vielleicht ein Zufall, in jedem Fall jedoch zeigt es, dass die fluide Identität, für die Prodger optiert, nicht auf Menschen begrenzt ist. Die Landschaft, die Natur, das Wasser, die Steine, die Pflanzen und die Tiere werden vielmehr, ebenso wie die Technik, zu Verbündeten im Prozess einer queeren Subjektivierung,

47 Vgl. den Beitrag von Henriette Gunkel in diesem Band. 
die sich nicht um ein Bezugssystem zentriert, sondern sich konstant in relationaler Bewegung zu anderen Bewegungen befindet. Obwohl es dabei spielerisch zugeht, ist daran nichts ironisch. Es geht um eine virtuelle Kraft, um eine Veränderung der Gegenwart und darin liegt das politische Moment dieser queeren Ästhetik. 
Astrid Deuber-Mankowsky, »Das iPhone als Medium einer queeren Ästhetik und fluiden Subjektivität. Charlotte Prodgers BRIDGIT «, in Queeres Kino / Queere Ästhetiken als Dokumentationen des Prekären, hg. v. Astrid Deuber-Mankowsky und Philipp Hanke, Cultural Inquiry, 22 (Berlin: ICI Berlin Press, 2021), S. 69-95 <https://doi.org/10.37050/ci-22_04>

\section{QUELLENANGABEN}

\section{BIBLIOGRAFIE}

Benjamin, Walter, Gesammelte Schriften, hg. v. Rolf Tiedemann und Hermann Schweppenhäuser, 7 Bde. (Frankfurt a. M.: Suhrkamp, 1972-91)

$\longrightarrow$ \Denkbilder $\ll$, in ders., Gesammelte Schriften, Iv (1972), S. 305-438

Butler, Judith, Antigones Verlangen: Verwandtschaft zwischen Leben und Tod, übers. v. Reiner Ansén (Frankfurt a. M.: Suhrkamp, 2001)

- Körper von Gewicht. Die diskursiven Grenzen des Geschlechts, übers. v. Karin Wördemann (Frankfurt a. M.: Suhrkamp, 1997)

— Psyche der Macht. Das Subjekt der Unterwerfung, übers. v. Reiner Ansén (Frankfurt a. M.: Suhrkamp, 1997)

_ Das Unbehagen der Geschlechter, übers. v. Kathrina Menke (Frankfurt a. M.: Suhrkamp, 1991)

Cope, Julian, The Modern Antiquarian: Pre-Millennial Odyssey through Megalithic Britain (London: Thorsons, 1998)

Deleuze, Gilles und Félix Guattari, Was ist Philosophie?, übers. v. Bernd Schwibs und Joseph Vogl (Frankfurt a. M.: Suhrkamp, 2000)

Deuber-Mankowsky, Astrid, Queeres Post-Cinema. Yael Bartana, Su Friedrich, Todd Haynes, Sharon Hayes (Berlin: August Verlag, 2017)

— $>>$ Für eine Maschine gibt es kein echtes Virtuelles<: Zur Kritik des Smartness Mandate mit Felwine Sarrs Afrotopia und Gilbert Simondons Philosophie der Technik «, in Digital/Rational, hg. v. Dieter Mersch und Katerina Krtilova (= Internationales Jahrbuch für Medienphilosophie, 6 (2020)), S. 131-45 <https://doi.org/10.1515/jbmp-2020-0007>

Drucker, Zackary, $\gg$ Sandy Stone on Living Among Lesbian Separatists as a Trans Woman in the 70 s $\ll$, Vice, 19. Dezember $2018<$ https://www.vice.com/en_us/article/zmd5k5/ sandy-stone-biography-transgender-history $>$ [Zugriff: 3. Juni 202 $\overline{1}$ ]

Film London, $\gg$ CHARLOTTE PRODGER - shortlisted artist profile - Film London Jarman Award 2017«, Vimeo, 23. August 2017 <https://vimeo.com/230803422> [Zugriff: 3. Juni 2021]

Krautkrämer, Florian, $\gg$ Revolution Uploaded. Un/Sichtbares im Handy-Dokumentarfilm «, Zeitschrift für Medienwissenschaft, 6.2 (2014), S. 113-26 <https://doi.org/10.25969/ mediarep/1257> 
Kulle, Daniel, »Innovation an den Rändern des Queer Cinema. Ästhetische Strategien des Queeren Experimentalfilms «, in Queer Cinema, hg. v. Dagmar Brunow und Simon Dickel (Mainz: Ventil, 2018), S. 226-44

Leaver-Yap, Mason, »Aesthetics and Anaesthetics in Charlotte Prodgers BRIDGIT $\ll$, in Bergen Kunsthall NO. 5 (2017) <http://hollybushgardens.co.uk/wp/wp-content/uploads/ CP_Mason-Leaver-Yap_Aesthetics-and-Anaesthetics.pdf> [Zugriff: 3. Juni 2021]

Simondon, Gilbert, Die Existenzweise technischer Objekte (Zürich: Diaphanes, 2012)

Tate Britain, »Charlotte Prodger | Turner Prize Winner 2018 | TateShots «, YouTube, 17. September 2018 <https://www.youtube.com/watch?v=AsVWk5DlbCE> [Zugriff: 3. Juni 2021]

\section{FILMOGRAFIE}

El Abuelo, Regie: Dino Dinco (Dino Dinco, 2004)

BRIDGIT, Regie: Charlotte Prodger (Hollybush Gardens, Charlotte Prodger, 2016)

High Flying Bird (Extension 765, Harper Road Films, 2019)

Nitrate Kisses, Regie: Barbara Hammer (Barbara Hammer, 1992)

SaF05, Regie: Charlotte Prodger (Hollybush Gardens, Charlotte Prodger, 2019)

Stoneymollan Trail, Regie: Charlotte Prodger (Hollybush Gardens, Charlotte Prodger, 2015)

Tender Fictions, Regie: Barbara Hammer (Barbara Hammer, 1996)

Unsane, Regie: Steven Soderbergh (Extension 765, New Regency Productions, 2018) 